\title{
Going for gold in COPD - a quality improvement project to reduce the inappropriate use of inhaled corticosteroids in the over $65 \mathrm{~s}$
}

\author{
Authors: Peter Robinson, ${ }^{A}$ Lucy Wicks ${ }^{B}$ and Andre Kuble ${ }^{C}$
}

\begin{abstract}
Introduction
Inhaled corticosteroids (ICS) are associated with increased rates of pneumonia, fracture, diabetes and mycobacterial infection in a dose dependent manner. They have limited short- and long-term benefit in chronic obstructive pulmonary disease, and cessation of steroids in selected patients has been proven safe. We observed that a large number of patients in the geriatric population were prescribed steroids, and set out to describe the incidence of steroid inhaler prescription among elderly care inpatients; we then sought to optimise respiratory care within an elderly care department in a central London teaching hospital.
\end{abstract}

\section{Method}

We conducted a retrospective analysis of patients discharged from a geriatric service over a 3-month period. Sequential discharges were assessed for the presence of inhaled therapies, the documented diagnosis and the proportion on inhaled therapies. After identifying high rates of inhaled therapy usage, we undertook a series of quality improvement interventions aimed at optimising respiratory care in these elderly patients. This included a guideline-based protocol for reviewing respiratory diagnoses and prescriptions.

\section{Results}

In the retrospective analysis, $67 / 297$ (22.5\%) patients were discharged on inhaled therapy; 55/67 (82\%) were discharged on inhaled steroids, of which 20/67 (36\%) were on high-dose equivalent ( $>1,000 \mu \mathrm{g}$ equivalent beclomethasone); 1/67 of these patients had evidence of inhaled therapy review during admission; and 15/55 of these patients were given a discharge diagnosis of pneumonia while only $6 / 55$ were admitted with an exacerbation of their chronic obstructive pulmonary disease. Our preliminary data suggest that a single-paged protocol aimed at improving adherence to best practice lead to practice change; dose modification has occurred in $50 \%$ of patients on inhaled therapy, leading to a reduction in steroid burden and an annual reduction in prescription costs of $€ 75$ per patient reviewed.

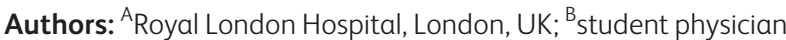
associate, Royal London Hospital, London, UK; ${ }^{C}$ Core medical trainee, Royal London Hospital, London, UK

\section{Conclusion}

By introducing measures to assist in the review and modification of inhaled therapies, we were able to change practice. The alteration in practice led to reduction in prescription costs, and a reduced burden of ICS. We propose that widespread encouragement of geriatrician-led respiratory review could lead to harm reduction and cost saving in the elderly.

\section{Conflicts of interest}

None declared. 